\title{
AVALIAÇÃO DA APRENDIZAGEM NO ESTÁGIO SUPERVISIONADO DE ENFERMAGEM EM SAÚDE COLETIVA
}

\author{
ASSESSMENT OF LEARNING IN THE SUPERVISED INTERNSHIP \\ IN NURSING IN COLLECTIVE HEALTH
}

\author{
EVALUACIÓN DE APRENDIZAJE DURANTE LA PRÁCTICA O \\ RESIDENCIA DE ENFERMERÍA EN SALUD COLECTIVA
}

\author{
Jameson Moreira Belém ${ }^{1}$ \\ Maria Juscinaide Henrique Alves ${ }^{2}$ \\ Glauberto da Silva Quirino ${ }^{3}$ \\ Evanira Rodrigues Maia ${ }^{4}$ \\ Maria do Socorro Vieira Lopes ${ }^{5}$ \\ Maria de Fátima Antero Sousa Machado ${ }^{6}$
}

Resumo Neste ensaio teórico-reflexivo, desenvolvemos uma discussão a respeito da avaliação da aprendizagem no processo de formação da assistência de Enfermagem em Saúde Coletiva no decorrer do estágio supervisionado. Dialogamos com autores da Saúde Coletiva e da avaliação da aprendizagem por competências, principalmente da medicina, que foram pioneiros nesse debate, empregando o modelo hierárquico-conceitual de avaliação de competências proposto por George Miller. Procuramos contribuir ao apresentar possibilidades de métodos, instrumentos e procedimentos de avaliação da aprendizagem em contextos somativos ou formativos, perspectivas e tendências da avaliação do processo de ensino-aprendizagem na área da Enfermagem. Constatamos que a avaliação por competências necessita ser mais discutida para estar alinhada às mudanças curriculares em curso e às demandas de formação de perfis profissionais em consonância com as necessidades e princípios do Sistema Único de Saúde.

Palavras-chave avaliação educacional; educação em enfermagem; estágio clínico; atenção primária à saúde; saúde coletiva.
Abstract In this thoughtful theoretical essay, we developed a discussion regarding the assessment of learning in the process of training for care in Nursing in Collective Health throughout the supervised internship. We had conversations with the authors of Collective Health and of the assessment of learning by competencies, mainly those involved in medicine, who were pioneers in this debate, using the hierarchical-conceptual model of assessment by competencies proposed by George Miller. We sought to make a contribution by presenting the possibilities of methods, tools and procedures for the assessment of learning in summative or formative contexts, and the perspectives and tendencies of the assessment of the teaching-learning process in the field of Nursing. We verified that the assessment by competencies needs to be more discussed in order to be in line with the ongoing syllabus changes and the demands for the training of professional profiles that is in accordance with the needs and principles of the Unified Health System (Sistema Único de Saúde, SUS, in the Portuguese acronym).

Keywords educational assessment; training in nursing; clinical internship; primary health care; collective health. 


\section{Introdução}

Ao se contextualizar o estágio supervisionado em Saúde Coletiva à formação em Enfermagem, observa-se uma ênfase na Atenção Primária à Saúde (APS). A APS, como nível de atenção do Sistema Único de Saúde (SUS), tem a Estratégia Saúde da Família (ESF) como eixo estruturante. Apesar das dificuldades em sua implementação e consolidação, a ESF tem contribuído para a (re)organização dos serviços, qualificação e humanização do atendimento, buscando assegurar a integralidade da atenção aos indivíduos, famílias e coletividades.

Em conformidade com o decreto n. 7.508, de junho de 2011, a APS insere-se como porta de entrada principal do SUS e ordenadora do cuidado em saúde, constituindo-se pilar edificador no contexto de mudança do paradigma no campo da Saúde Coletiva e da formação profissional para estar em consonância aos princípios do SUS. Para atender esses pressupostos, surge a necessidade de uma formação generalista, humanista, crítica e reflexiva, com perfil profissional qualificado para o exercício com base no rigor científico e intelectual, pautado em princípios éticos, senso de responsabilidade social e compromisso com a cidadania.

Esta formação pressupõe a inserção dos estudantes em diferentes cenários da prática profissional na qual os espaços e contextos de aprendizagem sejam capazes de superar o modelo biomédico, fragmentado e hospitalocêntrico de formação e atenção, o que possibilita ao graduando formar-se para atuar na promoção, proteção e recuperação da saúde.

Crescentes discussões têm evidenciado não apenas a importância de (re) orientação das práticas nos serviços de saúde, mas também a necessidade de pensar uma formação profissional que articule, desde seu início, ensino, serviço e comunidade, no intuito de desenvolver competências gerais e específicas, para atuação nesse cenário, que impliquem mudanças tanto nos modos de produção do trabalho em saúde quanto nos níveis de saúde da população (Benito et al., 2012; Colliselli et al., 2009; Fortuna et al., 2011; Vieira et al., 2016).

Desde as primeiras aproximações dos estudantes com a realidade de vida e saúde dos indivíduos, famílias e comunidades, deve-se possibilitar diversificados cenários de aprendizagem que sejam oportunos e significativos (Bollela e Machado, 2010a). Desse modo, o estágio supervisionado de Enfermagem em Saúde Coletiva constitui o ápice desse conjunto de experiências que devem ser vivenciadas na perspectiva do desenvolvimento de competências (atenção à saúde, tomada de decisões, comunicação, liderança, administração e gerenciamento e educação permanente) apoiadas em uma base concreta de conhecimentos associados à aquisição de habilidades, que fundamentem as atitudes e práticas, como previsto nas Diretrizes Curriculares Nacionais do curso de graduação em Enfermagem (Benito et al., 2012; Brasil, 2001). 
Disseminam-se práticas curriculares pouco sistematizadas, no decorrer dos cursos de graduação da saúde, que permitem apenas a inserção e o contato precoce dos alunos com a comunidade, mas que, devido à pontualidade e à falta de sistematização, caracterizam-se apenas como momentos reflexivos e observacionais (Bezerra et al., 2013). Efetiva-se, assim, a necessidade de estruturação de ações concretas e permanentes de inserção comunitária que perpetuem a visão do estudante sobre os atributos essenciais da atenção primária: acesso, longitudinalidade, coordenação, integralidade; e os atributos derivados: orientação familiar, orientação comunitária e competência cultural.

A rigor, as estratégias de ensino a serem desenvolvidas durante os estágios supervisionados em Saúde Coletiva dos cursos de graduação de Enfermagem devem primar por modelos que considerem a imersão do aluno em serviços de APS para vivenciar as práticas cotidianas na realidade do SUS.

Esta imersão permitiria ao graduando em Enfermagem não apenas conhecer os problemas de saúde de alta prevalência, mas participar ativa e efetivamente de ações rotineiras de prevenção, promoção, recuperação da saúde e avaliação do cenário de prática. Além disso, compreender contextos e fatores que podem interferir no processo saúde-doença e seus determinantes, buscar a integralidade das práticas de saúde, o trabalho em equipe, a intersetorialidade e interdisciplinaridade das ações e a integração com outros níveis de atenção do sistema de saúde (Campos e Forster, 2008).

O internato em Saúde Coletiva nos serviços de saúde deve contemplar diferentes dimensões da realidade social, econômica, política e cultural, mas a pertinência e a abrangência das práticas adotadas nestes cenários devem ser pensadas com base na problematização das experiências vividas no processo de ensino-aprendizagem. A ênfase no processo de planejamento dialógico deve permitir a construção de novos sentidos e inovações da prática do Estágio Supervisionado de Enfermagem em Saúde Coletiva (Rodrigues e Tavares, 2012).

O processo de ensino-aprendizagem deve pressupor a adoção de abordagens centradas no estudante, baseadas em problemas e orientadas à comunidade, viabilizadas por meio de metodologias ativas e da integração ensino-serviçocomunidade. Logo, devem concorrer para a formação de um profissional capaz de atuar de forma crítica e reflexiva nas necessidades sociais de saúde e de respondê-las segundo preceitos éticos, políticos e profissionais, adotando postura inovadora, consciente e responsável (Benito et al., 2012; Neves e Barros, 2014; Rodrigues, Tavares e Elias, 2014).

Com isso, espera-se que o estudante de Enfermagem, durante o estágio supervisionado, desenvolva competências gerais voltadas para a atenção à saúde, tomada de decisões, comunicação, liderança, administração, gerenciamento e educação permanente, as quais se manifestam apenas nas atividades práticas, em que podem ser avaliadas quanto ao seu desenvolvimento e suas implicações de uso (Benito et al., 2012). 
Considerando as tendências de modificações no processo ensino-aprendizagem em curso, pautadas na antecipação da inserção, diversificação dos cenários e uso de metodologias ativas, esforços devem ser dispensados para criar ambientes propícios à aprendizagem. Estes aspectos devem envolver os componentes cognitivos (o que aprender), afetivos (motivação para aprender) e metacognitivos (como aprender), bem como criar ambientes propícios à adaptação dos métodos de avaliação das competências a essa nova realidade (Amaral, Domingues e Bicudo-Zeferino, 2007).

Nesse sentido, esse ensaio teórico de caráter reflexivo ${ }^{7}$ se propõe a discorrer sobre a avaliação da aprendizagem no processo de formação da assistência de Enfermagem em Saúde Coletiva no decorrer do estágio supervisionado. A abordagem adotada pauta-se em informações atuais sobre o assunto em conformidade à literatura pertinente e relevante, congregando a interface entre a Saúde Coletiva e a formação em saúde por competências e as opiniões de pesquisadores das áreas.

$\mathrm{O}$ interesse pela temática provém da necessidade de formar competências gerais para atuação neste cenário de atenção e as incipientes formas de avaliação por competências que estão sendo instituídas no contexto das vivências e estágios no âmbito da atenção primária do SUS. Refletir o processo de ensino aprendizagem com foco na avaliação tem como pressuposto contribuir na discussão das metodologias do ensino superior dos cursos de graduação e pós-graduação da Enfermagem, bem como ampliar o debate sobre o ensino da Saúde Coletiva na formação dos profissionais em consenso com os processos de reformas e diretrizes curriculares dos cursos desta área.

\section{Avaliação de competências no estágio supervisionado e as perspectivas para a Enfermagem}

A crescente pressão da sociedade pela melhoria da qualidade assistencial e a conscientização da comunidade científica em relação ao grande número de profissionais que ingressam no mercado de trabalho com deficiências significativas de conhecimento e habilidades clínicas evidenciaram a necessidade de associar a matriz de competências aos objetivos de aprendizagem e aos métodos de avaliação (Bollela e Machado, 2010a).

O estudo de Letassy et al. (2015) identificou que existem déficits, inconsistências e desproporções na avaliação de conhecimentos, habilidades e atitudes relacionadas à Saúde Coletiva, porque são poucos ou incipientes os métodos, técnicas e procedimentos adotados na avaliação por competência, no entanto, as atuais estratégias encontram-se restritas à avaliação do desempenho.

A avaliação constitui um processo de julgamento consciente e, ao mesmo tempo, uma ação reflexiva, ética e dialógica, parte essencial do processo 
formativo que deve fornecer subsídios para a identificação de avanços e dificuldades no contexto do ensino e da aprendizagem. Deve ser desenvolvida de modo integrado, respeitar as diversidades, reconhecer que a aprendizagem ocorre em ritmos diferentes entre os estudantes, permitir que o aluno seja avaliado individualmente, segundo critérios pré-estabelecidos, sem influências e comparações entre desempenhos apresentados pelos aprendizes (Amaral, Domingues e Bicudo-Zeferino, 2007; Gontijo, Alvim e Lima, 2015; Neves e Barros, 2014).

A avaliação por competências vai além do simples ato de aprovar ou reprovar, deve mobilizar de forma articulada, contextualizada e integrada os domínios cognitivos, psicomotores e afetivos apresentados no desenvolvimento das atividades, devendo estar focada fundamentalmente no favorecimento da aprendizagem do estudante (Bollela e Machado, 2010a; Farias, 2015).

As práticas avaliativas devem sustentar-se em princípios pedagógicos capazes de mediar a formação de profissionais qualificados e comprometidos com a sua atuação. Logo, deve ser coerente com as diretrizes curriculares e o processo de ensino-aprendizagem, bem como acontecer de forma permanente, transparente, ética, democrática, participativa, critério-referenciada; levando em consideração o impacto educacional, a aceitabilidade, custo, confiabilidade, validade e envolver métodos somativos e formativos (Gontijo, Alvim e Lima, 2015; Neves e Barros, 2014).

Os métodos formativos compreendem práticas avaliativas contínuas que fornecem subsídios para o progresso da aprendizagem em curso; constituem fonte de regulação de todo o processo que permite o redirecionamento das ações adotadas por estudantes e docentes ou preceptores. No entanto, apenas será efetiva se o estudante for comunicado dos resultados, ou seja, se houver feedback. Em contrapartida, a avaliação somativa verifica as competências do estudante ao final de momentos específicos, e o alcance de objetivos previamente estabelecidos (Farias, 2015; Souza, 2012).

Para que permitam o efetivo acompanhamento e alcance dos objetivos educacionais e da aprendizagem, as avaliações formativas e somativas devem considerar critérios quantitativos (conteúdos mínimos que devem ser apresentados pelos estudantes); qualitativos (comportamentos desejados, quer sejam cognitivos, afetivos e psicomotores); e temporais (estabelecem os limites para a realização da avaliação da aquisição das competências) (Neves e Barros, 2014).

A escolha e desenvolvimento dos métodos de avaliação devem estar apoiados e baseados em uma estrutura tridimensional: primeiro, no conjunto de competências que serão avaliadas; segundo, nos objetivos da aprendizagem, considerando os quatro níveis desse processo: saber, saber como, demonstrar e fazer; e, terceiro, nos cinco níveis de desenvolvimento do aprendiz: iniciante, iniciante avançado, competente, proficiente e especialista (Amaral, Domingues e Bicudo-Zeferino, 2007; Bollela e Machado, 2010a). 
As experiências das escolas da área da saúde em relação à formação por competências, que aconteceram inicialmente na medicina, demonstraram a necessidade premente de mudanças curriculares neste e nos demais cursos que permitissem a sistematização da avaliação da aprendizagem por competências, considerando os avanços tecnológicos da área, o atual sistema de saúde, seu dinamismo e as conquistas sociais, notadamente a saúde como direito, e o controle social (Puccini, Sampaio e Batista, 2008).

Com base nestas transformações, os cursos da área da saúde foram estimulados a (re)pensar suas práticas de ensino e assistenciais na busca de currículos e modelos pedagógicos adequados aos padrões de metodologias ativas e à formação de profissionais de saúde de alto nível, capacitados para atender às necessidades da sociedade no contexto do SUS (Bollela e Machado, 2010a).

A prática regular de avaliação por competências é recente e ainda pouco difundida entre as instituições de ensino, sobretudo porque seu uso necessita de qualificação do corpo docente em métodos de avaliação adequados a este intento, bem como a adoção de políticas institucionais que estabeleçam critérios para a prática avaliativa. Ademais, a elaboração de instrumentos de avaliação de conhecimentos, habilidades e atitudes requer profundo refinamento das fases de planejamento, elaboração e execução, para que promovam a contínua adequação dos métodos de avaliação aos conteúdos propostos (Cyrino e Cyrino, 2012).

A avaliação deve fazer parte do processo de ensino-aprendizagem levando em consideração não somente o desempenho do aluno, mas, também, a atuação do docente ou preceptor, por meio do acompanhamento e de um processo continuado (Puccini, Sampaio e Batista, 2008). Muitos casos de aprendizagem deficiente resultam da inadequação na condução das atividades, no plano de ensino estabelecido ou método escolhido para avaliação. Portanto, torna-se necessário atentar para as estratégias selecionadas, o público em que serão implementadas, os objetivos a serem alcançados e a importância de rever o plano de ensino, numa dimensão de planejamento contínuo ao longo do curso, módulo ou disciplina a ser ministrada (Masetto, 2012).

Emerge o desafio de revisitar as estratégias de avaliação empreendidas para a formação profissional por meio da literatura nacional e internacional que busquem alinhar os pressupostos a serem adotados para um processo de avaliação condizente com as necessidades de saúde da população e da formação de competências na área da Enfermagem.

Algumas instituições de ensino em saúde têm incorporado práticas pedagógicas inovadoras em processos de avaliação da aprendizagem. O Quadro 1 apresenta alguns métodos, instrumentos e procedimentos de avaliação identificados na literatura nacional e internacional recente. 


\section{Quadro 1}

Possibilidades de métodos, instrumentos e procedimentos de avaliação da aprendizagem em contextos somativos e ou formativos de acordo com os níveis do modelo hierárquico-conceitual de avaliação de competências clínicas proposto por Miller (1990).

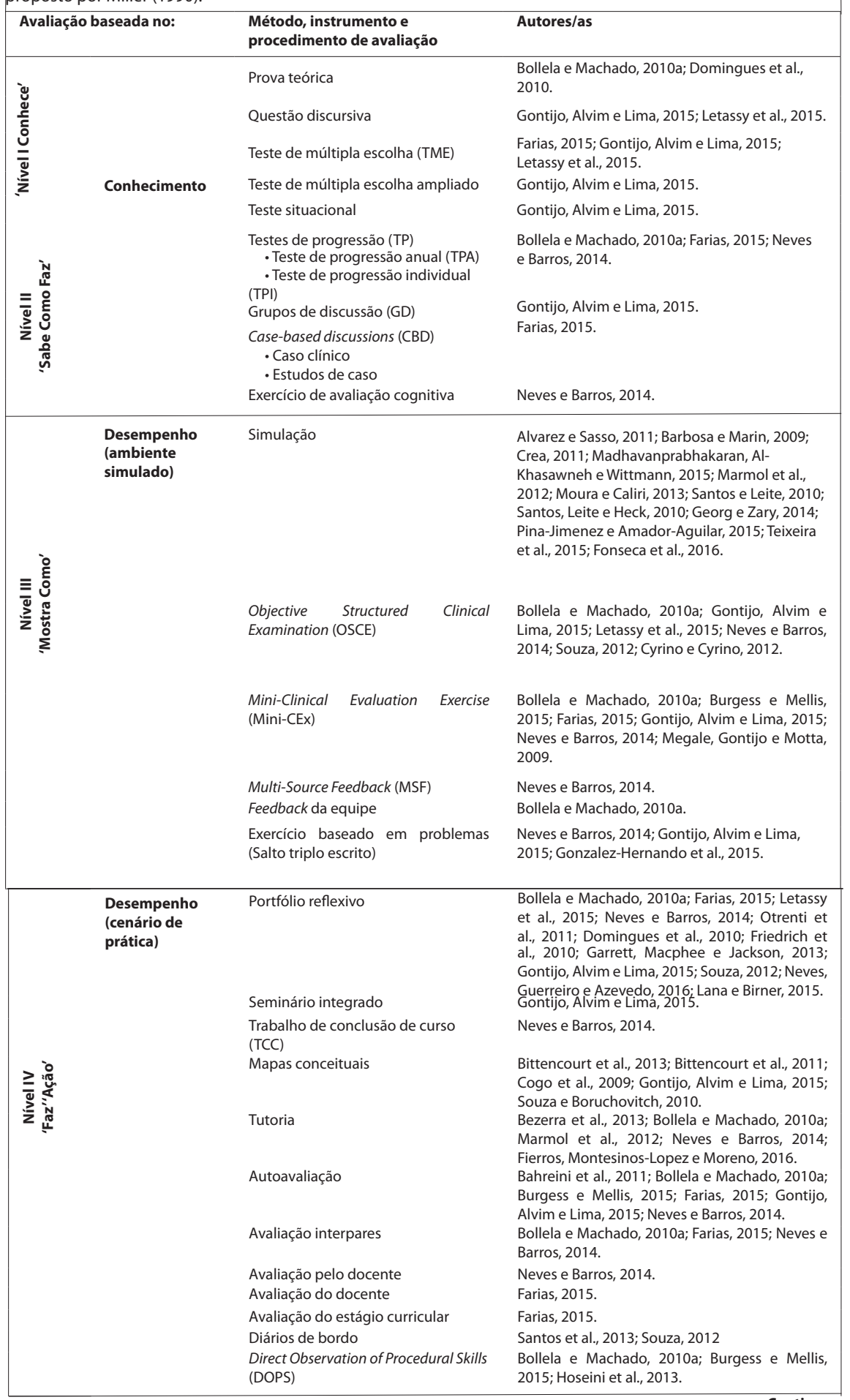




\begin{tabular}{|c|c|c|}
\hline \multicolumn{3}{|c|}{$\begin{array}{l}\text { Possibilidades de métodos, instrumentos e procedimentos de avaliação da aprendizagem em contextos somativos } \\
\text { e ou formativos de acordo com os níveis do modelo hierárquico-conceitual de avaliação de competências clínicas } \\
\text { proposto por Miller (1990). }\end{array}$} \\
\hline Avaliação baseada no: & $\begin{array}{l}\text { Método, instrumento e } \\
\text { procedimento de avaliação }\end{array}$ & Autores/as \\
\hline \multirow{12}{*}{$\begin{array}{l}\text { Desempenho } \\
\text { (cenário de } \\
\text { prática) }\end{array}$} & $\begin{array}{l}\text { Direct Observation of Procedural Skills } \\
\text { (DOPS) }\end{array}$ & $\begin{array}{l}\text { Bollela e Machado, 2010a; Burgess e Mellis, } \\
\text { 2015; Hoseini et al., } 2013 .\end{array}$ \\
\hline & $\begin{array}{l}\text { Avaliação baseada no local de } \\
\text { trabalho (ABLT) }\end{array}$ & Neves e Barros, 2014; Souza, 2012. \\
\hline & Avaliação diária do estudante & Farias, 2015; Neves e Barros, 2014. \\
\hline & $\begin{array}{l}\text { Avaliação sistematizada da prática } \\
\text { diária (ASPD) }\end{array}$ & Farias, 2015 \\
\hline & Conceito global & $\begin{array}{l}\text { Bollela e Machado, 2010a; Domingues, Amaral e } \\
\text { Bicudo-Zeferino, 2009; Domingues et al., 2010; } \\
\text { Gontijo, Alvim e Lima, } 2015 .\end{array}$ \\
\hline & $\begin{array}{l}\text { Exercício de investigação científica } \\
\text { (Projeto de intervenção - PI) }\end{array}$ & Neves e Barros, 2014 \\
\hline & $\begin{array}{l}\text { Exercício de avaliação da prática } \\
\text { profissional (EAPP) }\end{array}$ & Bahreini, et al., 2011; Neves e Barros, 2014. \\
\hline & Incidente crítico & Farias, 2015 \\
\hline & $\begin{array}{l}\text { Avaliação estruturada observacional } \\
\text { Acompanhamento oferecido pelos } \\
\text { preceptores }\end{array}$ & $\begin{array}{l}\text { Amaral, Domingues e Bicudo-Zeferino, } 2007 . \\
\text { Bezerra et al., 2013; Souza, } 2012 .\end{array}$ \\
\hline & Revisão prontuários & Bollela e Machado, 2010a. \\
\hline & $\begin{array}{l}\text { Avaliação Estruturada do } \\
\text { Atendimento Clínico (AEAC) }\end{array}$ & Domingues et al., 2010. \\
\hline & Handoff CEX & Horwitz et al., 2013. \\
\hline
\end{tabular}

Fonte: Elaborado pelos autores.

O desenvolvimento, implementação e sustentabilidade de uma ou mais técnicas de avaliação deve basear-se nas competências que se pretende avaliar, detalhadamente com base nos objetivos de aprendizagem. Logo, não existe método ineficaz. Cada um detém suas singularidades e indicações específicas. Cada um apresenta formas distintas de medir, averiguar e observar o desempenho do estudante, a depender do preparo do facilitador para utilizá-las e do cenário no qual serão aplicadas (Bollela e Machado, 2010a).

É necessário atentar-se à aplicabilidade de vários métodos para a valoração dos diferentes elementos que compõem as competências almejadas, uma vez que a combinação destes pode produzir resultados mais válidos e confiáveis (Domingues, Amaral e Bicudo-Zeferino, 2009).

Os métodos de avaliação podem valer-se de instrumentos estruturados (checklists) para auxiliarem durante as observações. Tais instrumentos consistem, em roteiros sistematizados que focalizam a atenção do avaliador para áreas, habilidades específicas e comportamentos a serem desenvolvidos, bem como oferecem elementos objetivos para fornecer feedback, reforçar pontos fortes e a corrigir fragilidades dos alunos (Amaral, Domingues e BicudoZeferino, 2007).

No entanto, as mesmas autoras destacam que estes checklists não devem apenas avaliar a presença ou ausência de características esperadas dos apren- 
dizes com base variáveis dicotômicas (Sim/Não). Esta configuração, apesar da praticidade, deixa de contemplar aspectos qualitativos (feedback e comparação de desempenho) e comportamentais que somente podem ser aferidos apuradamente através de processos contínuos e progressivos. Assim, sugerem que estes instrumentos devam conter uma escala de Likert, para diferenciar as nuances e gradações dos comportamentos esperados, evitando possíveis vieses no processo avaliativo.

Em contrapartida, a avaliação deve ocorrer de forma dialogada entre o estudante e seus instrutores, de modo que ambos possam assumir seus papéis no processo avaliativo e estejam comprometidos na construção de conhecimentos, habilidades e atitudes essenciais para a formação e qualificação do profissional enfermeiro (Farias, 2015). A implementação de qualquer ferramenta de avaliação depende basicamente da aceitação de alunos e docentes e ou preceptores, sendo que a satisfação desempenha um papel importante na aprendizagem e aquisição de habilidades e, portanto, deve ser considerada para se atingir os objetivos educacionais (Hoseini et al., 2013).

Uma avaliação efetiva deve estar ancorada em uma abordagem formativa, na qual o estudante esteja ciente a respeito do que se espera dele. Tal avaliação também deve ser equitativa com todos os participantes; provocar autorreflexão sobre o seu desempenho; fornecer direcionamento para os resultados esperados; adequar os métodos empregados aos objetivos de aprendizagem e oportunizar o feedback (Bollela e Machado, 2010a; Puccini, Sampaio e Batista, 2008)

O feedback, como componente central da avaliação e parte fundamental para efetivação da aprendizagem, permite informar ao discente sobre sua evolução e as necessidades e aspectos a serem melhorados, o que o motiva a envolverse nas atividades de ensino-aprendizagem adequadas (Burgess e Melli, 2015). O feedback tem capacidade para direcionar o processo de aprendizagem do estudante, reforçando comportamentos desejáveis. Por isso, deve representar um momento de crescimento no qual serão apresentadas alternativas, e não críticas para seu progresso (Burgess e Melli, 2015).

\section{Tendências da avaliação do processo de ensino-aprendizagem na}

\section{Enfermagem}

A literatura internacional dos últimos anos aponta que diversos cursos da área da saúde estão empregando uma variedade de métodos de avaliação, conforme disposto no Quadro 1, para capturar, avaliar o desempenho dos estudantes e obter resultados de aprendizagem mais eficazes (Bahreine et al., 2011; Crea, 2011; Garret, Macphee e Jackson, 2013; Horwitz et al., 2013; Hoseini et al., 2013; Letassy et al., 2015; Yahua e Watson, 2011). 
A Enfermagem tem se apropriado desses métodos, empregando-os para avaliação de conhecimentos, habilidades e atitudes nos diversos contextos de formação e atuação. Os mais comuns são os métodos baseados na observação, avaliação de supervisão, testes de conhecimento e habilidades, avaliação interpares, portfólios e autoavaliação. Mas há distinção entre eles de acordo com sua finalidade; enquanto alguns são mais centrados no desempenho da ação ('fazer'), outros são mais focados na cognição ('saber') (Bahreini et al., 2011; Domingues et al., 2010).

Dentre os métodos de avaliação utilizados pelas escolas internacionais de Enfermagem, destaca-se o portfólio reflexivo que representa um processo ativo de documentação das experiências, realizado pelos aprendizes durante determinado período de tempo, que envolve a coleta e organização de informações sobre as atividades desempenhadas e, principalmente, a reflexão quanto ao progresso e alcance dos objetivos de aprendizagem. Assim, por promover uma abordagem centrada no aluno, a aprendizagem ativa e a responsabilização individual, os portfólios têm sido incorporados na avaliação de competências dos estudantes quanto ao desenvolvimento de habilidades de pensamento crítico e tomada de decisão, devido à incorporação da reflexividade ao longo do processo (Amaral, Domingues e Bicudo-Zeferino, 2007; Garrett, Macphee e Jackson, 2013; Yanhua e Watson, 2011).

Em virtude da ampla variedade de tecnologias de informação e comunicação que têm surgido, existe uma demanda por instrumentos de avaliação eletrônicos, valorados e empregados para apoiar a avaliação prática formativa ou somativa do estudante como, por exemplo, os e-portfólios (Garrett, Macphee e Jackson, 2013).

O uso da simulação como metodologia de ensino tem sido empregado internacionalmente na formação de enfermeiros para desenvolver e avaliar competências antes de iniciar a prática propriamente dita; o que constitui método de familiarizar os alunos com os contextos reais de atuação, que reproduz as características e condições a serem avaliadas em um cenário simulado (Crea, 2011; Madhavanprabhakaran, Al-Khasawneh e Wittmann, 2015).

Ao considerar que estudantes da saúde, em especial de Enfermagem, têm dificuldade de compreender e aplicar seus conhecimentos teóricos em contextos práticos, o uso de pacientes simulados ou virtuais oferece um ambiente seguro e realista para se aprender, praticar e cometer erros sem contato direto com pacientes reais, o que favorece aprendizado ativo, permite repetições e facilita a correção imediata dos erros, sem risco para a segurança dos pacientes (Georg e Zary, 2014).

Ainda, podem apoiar os acadêmicos de Enfermagem na aprendizagem de conhecimentos científicos, integrar teoria e prática e promover o desenvolvimento de competências apoiadas em confiança, pensamento crítico para resolução de problemas, habilidades técnicas e não técnicas, tais como comu- 
nicação, comportamentos e trabalho em equipe (Madhavanprabhakaran, AlKhasawneh e Wittmann, 2015).

Existe, ainda, o uso do Objective Structured Clinical Examination (OSCE) na avaliação do desempenho e de aptidões clínicas, técnicas e práticas dos aprendizes de Enfermagem. Em situações delimitadas, baseadas em roteiros predefinidos que ocorrem ao longo de um conjunto de estações estruturadas como contextos clínicos simulados, nas quais, sob a forma de rodízio, as competências (saber como fazer) e o desempenho (mostrar como) dos alunos são avaliados pelos observadores ou pacientes simulados ou estandardizados por meio de checklists isolados ou combinados com notas ou conceitos (Amaral, Domingues e Bicudo-Zeferino, 2007; Ginsburg et al., 2015).

Assim como o Mini-Clinical Evaluation Exercise (Mini-CEX) é um instrumento de avaliação que permite realizar uma observação estruturada do desempenho prático dos estudantes por meio de guias de verificação ou checklists que contêm itens relacionados a atitudes e habilidades, nos quais se atribuem notas de avaliação com posterior feedback. O instrumento pode ser empregado enquanto o estudante realiza uma consulta objetiva e rápi$\mathrm{da}$, focada em determinada necessidade do paciente como, por exemplo, o histórico de enfermagem com ênfase na entrevista e exame físico (Amaral, Domingues e Bicudo-Zeferino, 2007; Horwitz et al., 2013; Megale, Gontijo e Motta, 2009).

Outros métodos para avaliação podem ser citados, como o Multi-source Feedback (MSF), o Direct Observation of Procedural Skills (DOPS), o Casebased Discussions (CBD), os diários de bordo e os mapas conceituais. Estes instrumentos de avaliação de competências e de autoavaliação têm sido testados quanto à viabilidade e validade como ferramentas úteis para prover aprendizagem, avaliação contínua e feedback e assim melhorar a qualidade das práticas nos cenários reais de formação (Horwitz et al., 2013).

A observação direta dos estagiários por meio dos Direct Observation of Procedural Skills (DOPS) constitui importante método de avaliação que emprega instrumentos ou checklists específicos para avaliação do desempenho de habilidades práticas ou clínicas, fornece feedback, otimiza a eficácia das aprendizagens experienciais e facilita a identificação das áreas de aprendizado a serem reforçadas (Amaral, Domingues e Bicudo-Zeferino, 2007; Hoseini et al., 2013).

Como métodos inovadores de avaliação clínica, os diários de bordo são usados por alunos para documentar observações e informações de desempenho, ajudando-os a se concentrarem em objetivos de aprendizagem (Hoseini et al., 2013). Ainda, os mapas conceituais despontam tanto como estratégias de ensino-aprendizagem quanto como ferramentas avaliativas capazes de criar possibilidades para a organização do conhecimento, promoção de 
experiências educativas pautadas na reflexão, compreensão e processamento profundo de informações, bem como criar possibilidades para o desenvolvimento da autorregulação, da metacognição e do aprender a aprender (Souza e Boruchovitch, 2010).

No Brasil, destacam-se experiências com alguns desses métodos que vêm sendo realizadas nos últimos anos, voltadas para o uso da simulação (Alvarez e Sasso, 2011; Barbosa e Marin, 2009; Marmol et al., 2012; Moura e Caliri, 2013; Santos e Leite, 2010; Santos, Leite e Heck, 2010; Teixeira et al., 2015), do portfólio reflexivo (Friedrich et al., 2010; Lana e Birner, 2015; Neves, Guerreiro e Azevedo, 2016; Otrenti et al. 2011), tutoria (Marmol et al., 2012), mapas conceituais (Bittencourt et al., 2011; Bittencourt et al., 2013; Cogo et al., 2009) e diários de bordo (Santos et al., 2013) na avaliação da aprendizagem na Enfermagem.

Em virtude da ampla variedade de métodos de avaliação disponíveis, sempre se faz necessário observar a pertinência de cada um, analisar as etapas e estratégias que devem ser idealmente desenvolvidas, seus atributos, particularidades, limitações, potencialidades, vantagens e desvantagens educacionais para que estejam alinhadas aos objetivos de aprendizagem propostos e em consonância às competências almejadas.

Apesar de existir uma demanda por novas formas de avaliação da aprendizagem, o uso de métodos avaliativos atualmente ainda se encontra, por vezes, centrado em modelos tradicionais de aferição da quantidade assuntos assimilados. Portanto, insuficientes para avaliar a aprendizagem do estudante (Bollela e Machado, 2010a). Por outro lado, observa-se que o emprego desses métodos deve caminhar para o contexto da atenção primária à saúde, sendo incorporados nos estágios supervisionados de Saúde Coletiva, visto que se encontram, na maioria das vezes, restritos a contextos clínicos e ou hospitalares.

Dentre os modelos de avaliação em Saúde Coletiva, observa-se a ausência de estratégias integralizadoras e de linhas de ação compatíveis com a realidade de saúde, bem como a desarticulação entre a elaboração da programação e do processo de supervisão do aluno e os profissionais dos serviços de saúde. Sem contar que o acompanhamento é realizado, com frequência, por preceptores alheios tanto ao planejamento quanto à realidade do campo de estágio, o que implica em negociações de saber e poder na condução desse agir coletivo que podem comprometer o processo de ensino-aprendizagem (Rodrigues, Tavares e Elias, 2014).

O estágio supervisionado tem se configurado como espaço em que predominam a reprodução, a repetição do instituído e a exclusão como regra (Fortuna et al., 2011). Não obstante, ainda existem dificuldades para assegurar uma adequada interação entre os conhecimentos teóricos adquiridos e as práticas inseridas nos cenários de atuação (Cyrino e Cyrino, 2012). 
Reafirma-se, assim, a necessidade imperiosa de se pensar e fazer o ensino em saúde sob uma nova concepção voltada para a produção e articulação de saberes, e a necessidade não apenas de revisar, atualizar e operar as disciplinas que compõem o currículo de graduação, mas de (re)formular estratégias educacionais para o ensino no Estágio Supervisionado (Rodrigues, Tavares e Elias, 2014).

\section{Considerações finais}

Este artigo teórico-reflexivo introduz discussões importantes para a Enfermagem e para a área de ensino, ao explorar um referencial teórico e instrumentos de avaliação que contribuem para a construção de uma Enfermagem diferenciada e em consonância com o contexto atual de saúde e educação, com base em referências bibliográficas relevantes e atuais, que não se encerram por si mesmas, visto que as reflexões podem ser acrescidas de outras que se pautaram nos referenciais apontados.

Acresce-se que a avaliação do processo de ensino-aprendizagem é uma temática que ainda necessita ser bastante explorada, tendo em vista que poucas instituições adotam a avaliação por competência nos seus projetos políticos pedagógicos.

Bollela e Machado (2010b) apontam que, apesar de as instituições de ensino estarem implementando mudanças curriculares que empregam metodologias inovadoras, ainda existem lacunas e descompassos entre as práticas pedagógicas dos primeiros anos de curso e aquelas observadas durante os estágios supervisionados. Esta situação demanda currículos claramente definidos, baseados em competências com docentes e preceptores capacitados, para que se possa, segundo os autores, construir a ponte (bridging the gap) que ajudaria a diminuir a distância entre esses dois importantes momentos do processo de ensino-aprendizagem e consequentemente qualificar a formação dos profissionais.

Surgem como imprescindíveis as reformulações curriculares nos cursos de graduação em saúde e o (re)pensar das práticas pedagógicas para que estejam em consonância com as demandas de formação de profissionais no contexto das Diretrizes Curriculares, capazes de entender e atender as demandas do SUS e atuar em seus diferentes contextos políticos, econômicos, históricos, culturais e sociais.

Este percurso, certamente, não representa tarefa fácil, pois envolve mudanças de paradigma, concepções e posturas em relação ao processo de ensino-aprendizagem, mas, diante das transformações que vêm ocorrendo nos últimos anos, podem-se pressagiar mudanças, o que sinaliza para um processo em permanente discussão e construção. 


\section{Agradecimentos}

Agradecemos à Fundação Cearense de Apoio ao Desenvolvimento Científico e Tecnológico pelo apoio financeiro recebido, em forma de concessão de bolsa de mestrado ao primeiro autor.

\section{Colaboradores}

Jameson Moreira Belém e Maria Juscinaide Henrique Alves foram responsáveis pela concepção do projeto, análise e interpretação dos dados, redação do artigo e revisão crítica. Glauberto da Silva Quirino, Evanira Rodrigues Maia, Maria do Socorro Vieira Lopes, Maria de Fátima Antero Sousa Machado participaram da análise e interpretação dos dados, redação do artigo e revisão crítica. Todos aprovaram a versão final a ser publicada. Não há conflito de interesses.

Resumen En este ensayo teórico reflexivo, desarrollamos una discusión acerca de la evaluación de aprendizaje en el proceso de formación de asistencia de Enfermería en Salud Colectiva durante la práctica o residencia. Dialogamos con autores sobre Salud Colectiva y la evaluación de aprendizaje por competencias, principalmente de la medicina, que fueron pioneros en este debate, empleando el modelo jerárquico-conceptual de evaluación de competencias propuesto por George Miller. Buscamos contribuir al presentar posibilidades de métodos, instrumentos y procedimientos de evaluación de aprendizaje en contextos sumativos o formativos, perspectivas y tendencias de la evaluación del proceso de enseñanza-aprendizaje en el área de Enfermería. Constatamos que la evaluación por competencias necesita ser más discutida para alinearse a los cambios curriculares en curso y a las demandas de formación de perfiles profesionales de acuerdo a las necesidades y principios del Sistema Único de Salud.

Palabras clave evaluación educativa; educación en enfermería; práctica profesional; atención primaria de la salud; salud colectiva. 


\section{Notas}

${ }^{1}$ Universidade Regional do Cariri, Programa de Pós-Graduação em Enfermagem, Crato, Ceará, Brasil.

$<$ jam.ex@hotmail.com>

Correspondência: Universidade Regional do Cariri, Centro de Ciências Biológicas e da Saúde, Departamento de Enfermagem, Rua Coronel Antônio Luiz, 1.161, Pimenta, CEP 63105-000, Crato, Ceará, Brasil.

${ }^{2}$ Universidade Regional do Cariri, Programa de Pós-Graduação em Enfermagem, Crato, Ceará, Brasil.

$<$ juscinaidehenrique@hotmail.com>

${ }^{3}$ Universidade Regional do Cariri, Programa de Pós-Graduação em Enfermagem, Crato, Ceará, Brasil.

<glauberto.quirino@urca.br>

${ }^{4}$ Universidade Regional do Cariri, Programa de Pós-Graduação em Enfermagem, Crato, Ceará, Brasil.

<evanira.maia@urca.br>

${ }^{5}$ Universidade Regional do Cariri, Programa de Pós-Graduação em Enfermagem, Crato, Ceará, Brasil.

$<$ socorrovieira@hotmail.com>

${ }^{6}$ Universidade Regional do Cariri, Programa de Pós-Graduação em Enfermagem, Crato, Ceará, Brasil.

$<$ fatimaantero@uol.com.br>

${ }^{7}$ Artigo elaborado na disciplina de Metodologia do Ensino Superior do Curso de Mestrado Acadêmico em Enfermagem do Programa de Pós-Graduação em Enfermagem da Universidade Regional do Cariri. 


\section{Referências}

ALVAREZ, Ana G.; SASSO, Grace T. M. D. Aplicação de objeto virtual de aprendizagem, para avaliação simulada de dor aguda, em estudantes de enfermagem. Revista LatinoAmericana de Enfermagem, Ribeirão Preto, São Paulo, v. 19, n. 2, p. 229-237, mar.-abr. 2011.

AMARAL, Eliana; DOMINGUES, Rosângela C. L.; BICUDO-ZEFERINO, Angélica M. Avaliando competência clínica: o método de avaliação estruturada observacional. Revista Brasileira de Educação Médica, Brasília, Distrito Federal, v. 31, n. 3, p. 287-290, 2007.

BAHREINI, Masoud et al. Comparison of head nurses and practicing nurses in nurse competence assessment. Iranian Journal of Nursing and Midwifery Research, Isfahan, v. 16, n. 3, p. 227-234, 2011.

BARBOSA, Sayonara F. F; MARIN, Heimar F. Simulação baseada na web: uma ferramenta para o ensino de enfermagem em terapia intensiva. Revista Latino-Americana de Enfermagem, Ribeirão Preto, v. 17, n.1, p. 7-13, jan. fev. 2009.

BENITO, Gladys A. V. et al. Desenvolvimento de competências gerais durante o estágio supervisionado. Revista Brasileira de Enfermagem, Brasília, Distrito Federal, v. 65, n. 1, p. 172178, jan.-fev. 2012.

BEZERRA, Aline P. S. et al. A percepção do graduando em Saúde Coletiva sobre o estágio supervisionado. Tempus Actas de Saúde Coletiva, Brasília, v. 7, n. 3, p. 115-127, 2013.

BITTENCOURT, Greicy K. G. D. et al. Aplicação de mapa conceitual para identificação de diagnósticos de enfermagem. Revista Brasileira de Enfermagem, Brasília, Distrito Federal, v. 64, n. 5, p. 963-967, set.-out. 2011.

BITTENCOURT, Greicy K. G. D. et al. Mapas conceituais no ensino de pós-graduação em Enfermagem: relato de experiência. Revista Gaúcha de Enfermagem, Porto Alegre, Rio Grande do Sul, v. 34, n. 2, p. 172-176, 2013.
BOLLELA, Valdes R.; MACHADO, José L. M. Internato baseado em competências: "Bridging the gaps". Minas Gerais: Editora Medvance, 2010a.

BOLLELA, Valdes R.; MACHADO, José L. M. O currículo por competências e sua relação com as diretrizes curriculares nacionais para a graduação em Medicina. Science in Health, São Paulo, São Paulo, v. 1, n. 2, p. 126-42, maio-ago. 2010b.

BRASIL. Ministério da Educação. Resolução CNE/CES n. 3, de 7 de novembro de 2001. Institui Diretrizes Curriculares Nacionais do Curso de Graduação em Enfermagem. Diário Oficial da União, Poder Executivo, Brasília, 9 nov. 2001, Seção 1, p.1-6.

BURGESS, Annette; MELLIS, Craig. Feedback and assessment for clinical placements: achieving the right balance. Advances in Medical Education and Practice, v. 6, p. 373-381, 2015.

CAMPOS, Maria A. F.; FORSTER, Aldaísa C. Percepção e avaliação dos alunos do curso de Medicina de uma escola médica pública sobre a importância do estágio em Saúde da Família na sua formação. Revista Brasileira de Educação Médica, Brasília, Distrito Federal, v. 32, n. 1, p. 83-89, 2008.

COGO, Ana L. P. et al. Avaliação de mapas conceituais elaborados por estudantes de Enfermagem com o apoio de software. Texto \& Contexto Enfermagem, Florianópolis, Santa Catarina, v. 18, n. 3, p. 482-488, jul.-set. 2009.

COLLISELLI, Liane et al. Estágio curricular supervisionado: diversificando cenários e fortalecendo a interação ensino-serviço. Revista Brasileira de Enfermagem, Brasília, Distrito Federal, v. 62, n. 6, p. 932-937, nov.-dez. 2009.

CREA, Kathryn A. Practice skill development through the use of human patient simulation. The American Journal of Pharmaceutical Education, Lincoln, v. 75, n. 9, p. 1-8, nov. 2011. 
CYRINO, Eliana G.; CYRINO, Antônio P. A avaliação de habilidades em saúde coletiva no internato e na prova de residência médica na Faculdade de Medicina de Botucatu - Unesp. In: TIBÉRIO, Iolanda F. L. C. et al (org.). Avaliação prática de habilidades em medicina. 1. ed. São Paulo: Editora Atheneu, 2012. p. 163-172.

DOMINGUES, Rosângela C. L. et al. Competência clínica de alunos de Medicina em estágio clínico: comparação entre métodos de avaliação. Revista Brasileira de Educação Médica, Brasília, v. 34, n. 1, p. 124-131, 2010.

DOMINGUES, Rosângela C. L.; AMARAL, Eliana; BICUDO-ZEFERINO, Angélica $M$. Conceito global: um método de avaliação de competência clínica. Revista Brasileira de Educação Médica, Brasília, v. 33, n. 1, p. 148-151, 2009.

FARIAS, Lindalva M. R. Manual do estágio curricular do curso de graduação em Enfermagem. Brasília: Fundação de Ensino e Pesquisa em Ciências da Saúde/Escola Superior de Ciências da Saúde, 2015. 55 p.

FIERROS, Genoveva A.; MONTESINOS-LÓPEZ, Osval A.; MORENO, Noemí A. Validation of an instrument to measure tutor performance in promoting self-directed learning by using confirmatory factor analysis. Investigación y Educación en Enfermería, Medellín, v. 34, n. 1, p. 74-83, abr. 2016.

FONSECA, Luciana M. M. et al. Computer and laboratory simulation in the teaching of neonatal nursing: innovation and impact on learning. Revista Latino-Americana de Enfermagem, Ribeirão Preto, v. 24, e2808, 2016.

FORTUNA, Cinira M. et al. A pesquisa e a articulação ensino-serviço na consolidação do Sistema Único de Saúde. Revista da Escola de Enfermagem da USP, São Paulo, v. 45, núm. esp. 2, p. 1.696-1.700, 2011.

FRIEDRICH, Denise B. C. et al. O portfólio como avaliação: análise de sua utilização na graduação de enfermagem. Revista Latino-
Americana de Enfermagem, São Paulo, v. 18, n. 6, p. 1.123-1.130, nov.-dez. 2010.

GARRETT, Bernard M.; MACPHEE, Maura; JACKSON, Cathryn. Evaluation of an eportfolio for the assessment of clinical competence in a baccalaureate nursing program. Nurse Education Today, Edinburgh, v. 33, n. 10, p. 1.207-1.213, out. 2013.

GEORG, Carina; ZARY, Nabil. Web-based virtual patients in nursing Education: development and validation of theory-anchored design and activity models. Journal of Medical Internet Research, Pittsburgh, v. 16, n. 4, abr. 2014.

GINSBURG, Liane R. et al. Development and testing of an objective structured clinical exam (OSCE) to assess socio-cultural dimensions of patient safety competency. BMJ Quality \& Safety, London, v. 24, p. 179-181, 2015.

GONZALEZ-HERNANDO, C. et al. Evaluación por los estudiantes al tutor de enfermería en el contexto del aprendizaje basado en problemas. Enfermería Universitaria, Ciudad de México, v. 12 , n. 3, p. 110-115, set. 2015 .

GONTIJO, Eliane D.; ALVIM, Cristina G.; LIMA, Maria E. C. C. Manual de avaliação da aprendizagem do curso de graduação em Medicina. Revista Docência do Ensino Superior, Belo Horizonte, v. 5, n. 1, p. 205-326, abr. 2015 .

HORWITZ, Leora I. et al. Validation of a handoff assessment tool: the Handoff CEX. Journal of Clinical Nursing, Oxford, v. 22, p. 1.477-1.486, maio 2013.

HOSEINI, Bibi L. et al. Comparison of midwifery students' satisfaction with direct observation of procedural skills and current methods in evaluation of procedural skills in Mashhad Nursing and Midwifery School. Iranian Journal of Nursing and Midwifery Research, Isfahan, v. 18, n. 2, p. 94-100, mar.-abr., 2013.

LANA, Letice D.; BIRNER, Juceline A. Um relato de caso sobre a construção e elaboração do portfólio como metodologia avaliativa de 
aprendizagem. Ciencia y Enfermería, Concepción, v. 21, n. 3, p. 101-112, 2015.

LETASSY, Nancy A. et al. A progressive, collaborative process to improve a curriculum and define an assessment program. The American Journal of Pharmaceutical Education, Lincoln, v. 79, n. 4, p.1-5, maio 2015.

MADHAVANPRABHAKARAN, Girija; ALKHASAWNEH, Esra; WITTMANN, Lane. Perceived benefits of pre-clinical simulation-based training on clinical learning outcomes among Omani undergraduate nursing students. Sultan Qaboos University Medical Journal, Muscat, v. 15, n. 1, p. 105-111, fev. 2015.

MARMOL, Milene T. et al. Curativo de cateter central em simulador: efeito da presença do tutor ou da aprendizagem autoinstrucional. Revista Latino-Americana de Enfermagem, Ribeirão Preto, v. 20, n. 6, p. 1134-1141, nov.-dez. 2012.

MASETTO, Marcos T. Competência pedagógica do professor universitário. 2 ed. São Paulo: Summus, 2012. 194 p.

MEGALE, Luiz; GONTIJO, Eliana D.; MOTTA, Joaquim A. C. Avaliação de competência clínica em estudantes de Medicina pelo miniexercício clínico avaliativo (Miniex). Revista Brasileira de Educação Médica, Brasília, v. 33, n. 2, p. 166-175, 2009.

MILLER, George E. The assessment of clinical skills/competence/performance. Academic Medicine, Philadelphia, v. 65, supl. 9, p. 63-7, 1990.

MOURA, Elaine C. C.; CALIRI, Maria H. L. Simulação para desenvolvimento da competência clínica de avaliação de risco para úlcera por pressão. Acta Paulista de Enfermagem, São Paulo, v. 26, n. 4, p. 369-375, 2013.

NEVES, Rinaldo S.; BARROS, Ângela F. (org.). Manual de avaliação do curso de graduação em Enfermagem. Brasília: Fundação de Ensino e Pesquisa em Ciências da Saúde; Escola Superior de Ciências da Saúde, 2014. 41 p.
NEVES, Andressa S. C.; GUERREIRO, José M. A.; AZEVEDO, Gisele R. Avaliando o portfólio do estudante: uma contribuição para o processo de ensino-aprendizagem. Avaliação: Revista da Avaliação da Educação Superior, Sorocaba, v. 21, n. 1, p. 199-220, mar. 2016.

OTRENTI, Eloá et al. Portfólio reflexivo como método de avaliação na residência de gerência de serviços de enfermagem. Semina: Ciências Biológicas e da Saúde, Londrina, v. 32, n. 1, p. 41-46, jan.-jun. 2011.

PINA-JIMENEZ, Irma; AMADOR-AGUILAR, Rocío. La enseñanza de la enfermería con simuladores, consideraciones teórico-pedagógicas para perfilar un modelo didáctico. Enfermería Universitária, Ciudad de México, v. 12, n. 3, p. 152-159, set. 2015.

PUCCINI, Rosana F.; SAMPAIO, Lucia O.; BATISTA, Nildo A. (org.). A formação médica na Unifesp: excelência e compromisso social. São Paulo: Editora Unifesp, 2008. 312 p.

RODRIGUES, Lília M. S.; TAVARES, Claudia M. M. Estágio supervisionado de Enfermagem na atenção básica: o planejamento dialógico como dispositivo do processo ensino-aprendizagem. Revista da Rede de Enfermagem do Nordeste, Fortaleza, v. 13, n. 5, p. 1.075-1.083, 2012.

RODRIGUES, Lília M. S.; TAVARES, Claudia M. M.; ELIAS, Andrea D. S. Interação, ensino e serviço de Saúde para o desenvolvimento do estágio supervisionado em Enfermagem na atenção básica. Journal of Research: Fundamental Care Online, Rio de janeiro, v. 6, n.1, p. 357-363, jan.-mar. 2014.

SANTOS, José L. G. et al. Diário de bordo: experiência de ensino-aprendizagem de Gestão e Gerenciamento em Saúde e Enfermagem. Revista da Rede de Enfermagem do Nordeste, Fortaleza, v. 14, n. 4, p. 854-863, 2013.

SANTOS, Mateus C.; LEITE, Maria C. L. A avaliação das aprendizagens na prática da simulação em Enfermagem como feedback de ensino. Revista Gaúcha de Enfermagem, Porto Alegre, v. 31, n.3, p. 552-556, set. 2010. 
SANTOS, Mateus C.; LEITE, Maria C. L.; HECK, Rita M. Recontextualização da simulação clínica em Enfermagem baseada em Basil Bernstein: semiologia da prática pedagógica. Revista Gaúcha de Enfermagem, Porto Alegre, v. 31, n. 4, p. 746-752, dez. 2010.

SOUZA, Nadia A.; BORUCHOVITCH, Evely. Mapas conceituais: estratégia de ensino/aprendizagem e ferramenta avaliativa. Educação em Revista, Belo Horizonte, v. 26, n. 3, p. 195-218, dez. 2010.

SOUZA, Ruy G. S. Atributos fundamentais dos procedimentos de avaliação. In: TIBÉRIO, Iolanda F. L. C. et al. Avaliação prática de habilidades em Medicina. São Paulo: Editora Atheneu, 2012. p. 2-11.

TEIXEIRA, Carla R. S. et al. Avaliação dos estudantes de Enfermagem sobre a aprendi- zagem com a simulação clínica. Revista Brasileira de Enfermagem, Brasília, v. 68, n. 2, p. 311-319, abr. 2015.

VIEIRA, Leila M. et al. Formação profissional e integração com a rede básica de saúde. Trabalho, Educação e Saúde, Rio de Janeiro, v. 14, n. 1, p. 293-304, jan.-abr. 2016.

YANHUA, Chen; WATSON, Roger. A review of clinical competence assessment in nursing. Nurse Education Today, Edinburgh, v. 31, n. 8 , p. $832-836$, nov. 2011.

Recebido em 30/03/2016

Aprovado em 20/05/2017 